\title{
The Relationships Among Occupational Safety Climate, Patient Safety Climate, and Safety Performance Based on Structural Equation Modeling
}

\author{
Hamed Aghaei ${ }^{1,2}$, Zahra Sadat Asadi ${ }^{1}$, Mostafa Mirzaei Aliabadii ${ }^{3}$, Hassan Ahmadinia ${ }^{4}$ \\ ${ }^{1}$ Department of Community Medicine, School of Medicine, Aja University of Medical Sciences, Tehran, Iran; ${ }^{2}$ Department of Occupational Health \\ Engineering, School of Health, Arak University of Medical Sciences, Arak, Iran; ${ }^{3}$ Center of Excellence for Occupational Health (CEOH) and \\ Occupational Health and Safety Research Center, Hamadan University of Medical Sciences, Hamadan, Iran; ${ }^{4}$ Department of Epidemiology and \\ Biostatistics, Medical School, Rafsanjan University of Medical Sciences, Rafsanjan, Iran
}

Objectives: The aim of the present study was to investigate the relationships among hospital safety climate, patient safety climate, and safety outcomes among nurses.

Methods: In the current cross-sectional study, the occupational safety climate, patient safety climate, and safety performance of nurses were measured using several questionnaires. Structural equation modeling was applied to test the relationships among occupational safety climate, patient safety climate, and safety performance.

Results: A total of 211 nurses participated in this study. Over half of them were female (57.0\%). The age of the participants tended to be between 20 years and 30 years old (55.5\%), and slightly more than half had less than 5 years of work experience (51.5\%). The maximum and minimum scores of occupational safety climate dimensions were found for reporting of errors and cumulative fatigue, respectively. Among the dimensions of patient safety climate, non-punitive response to errors had the highest mean score, and manager expectations and actions promoting patient safety had the lowest mean score. The correlation coefficient for the relationship between occupational safety climate and patient safety climate was $0.63(p<0.05)$. Occupational safety climate and patient safety climate also showed significant correlations with safety performance.

Conclusions: Close correlations were found among occupational safety climate, patient safety climate, and nurses' safety performance. Therefore, improving both the occupational and patient safety climate can improve nurses' safety performance, consequently decreasing occupational and patient-related adverse outcomes in healthcare units.

Key words: Safety climate, Statistical model, Safety behavior, Nurses

\section{INTRODUCTION}

Received: July 24, 2020 Accepted: October 3, 2020

Corresponding author: Zahra Sadat Asadi, PhD Department of Community Medicine, School of Medicine, Aja

University of Medical Sciences, Tehran 1411718541, Iran

E-mail: 2006.asadi@gmail.com

This is an Open Access article distributed under the terms of the Creative Commons Attribution Non-Commercial License (https://creativecommons.org/licenses/by$\mathrm{nc} / 4.0 /$ ) which permits unrestricted non-commercial use, distribution, and reproduction in any medium, provided the original work is properly cited.
Hospitals and healthcare units are classified as among the 5 most hazardous occupational environments [1]. Based on the United States Bureau of Labor Statistics, nursing accounts for the 13th highest rate of accidents in the United States, and is classified as the occupation with the seventh highest number of working days lost due to accidents [2]. Adverse events due to unsafe care of patients in healthcare units are also an 
important concern [3]. Therefore, it is essential to pay particular attention to problems related to the safety and health of healthcare professionals and the safety of patients, which are critical indicators of the quality of healthcare units.

\section{Safety Climate}

The concept of the safety climate was first introduced by Zohar [4] in 1980. The safety climate refers to workers' perceptions of safety and influences workers' motivation to behave in a safe manner [5]. The safety climate depends on time and place and reflects the current situation of the organization; hence, it is relatively unstable and changes based on the situation of the organization [6].

\section{Patient Safety Climate}

Patient safety is considered to be the most crucial parameter in the quality of healthcare units [7]. Adverse accidents leading to injuries in patients in healthcare units indicate that more attention should be paid to patient safety [8]. A study by the National Health Service found that at least $25 \%$ of medical staff reported experiencing an error that resulted in patient injury [9]. Improvements in patient safety can be attributed to the patient safety climate. The patient safety climate in healthcare units includes communication based on trust, appropriate information flow, a shared understanding of safety, and a commitment to management and leadership [10].

\section{Safety Performance}

Studies have shown that more than $85 \%$ of accidents occur due to workers' safety performance [11]. Safety performance is the actual behavior that individuals perform at work [12], and consists of safety participation and safety compliance. Safety compliance includes activities that individuals should carry out to improve their safety. Safety participation includes behaviors that do not play a direct role in providing safety, but help to create conditions that enhance safety in the organization and facilitate the achievement of the organization's safety goals and programs [13].

\section{Relationships Among Safety Climate, Patient Safety Climate, and Safety Performance}

Unsafe behavior of workers is a major cause of workplace accidents. Many studies [14-16] have reported that safety climate is an essential predictor of safety performance. A highlevel of safety climate in an organization improves workers' safety performance and reduces the risk of adverse outcomes for both workers and patients. The safety climate may have a significant impact on the priorities and performance of workers, which may affect the safety of both patients and workers [17]. Previous studies have shown that in healthcare units with an excellent patient safety climate, both staff and patients experienced a low rate of occupational and medical accidents [9]. According to Flin [18]'s study, the occupational safety climate and patient safety climate affect unsafe behavior, and hence unsafe behavior can affect human errors and pose a risk to staff and patients.

Prior studies have investigated the relationships among patient safety climate, occupational safety climate, and safety performance $[19,20]$. However, no comprehensive study has yet investigated the relationships among these factors, especially in developing countries such as Iran. Therefore, this study aimed to evaluate the relationships among occupational safety climate, patient safety climate, and safety performance in Iranian healthcare units. In the current study, the following hypotheses were tested:

Hypothesis 1: Occupational safety climate has a positive relationship with safety performance.

Hypothesis 2: Patient safety climate has a positive relationship with safety performance.

Hypothesis 3: Occupational safety climate and patient safety climate are related to each other.

The designed hypothetical model is illustrated in Figure 1.

\section{METHODS}

This cross-sectional study was conducted at 2 large hospitals in the west of Iran in 2019. The participants included all nurses with at least 1 year of work experience.

\section{Data Collection}

Data collection was conducted based on 4 questionnaires, including occupational safety climate, patient safety climate, safety performance, and demographic and job characteristics. The last questionnaire consisted of 9 questions about job characteristics and personal information.

\section{Occupational safety climate}

The occupational safety climate of the nurses was evaluated using the questionnaire developed by Zarei et al. [21]. This questionnaire measures 7 dimensions of occupational safety climate 
Journal of

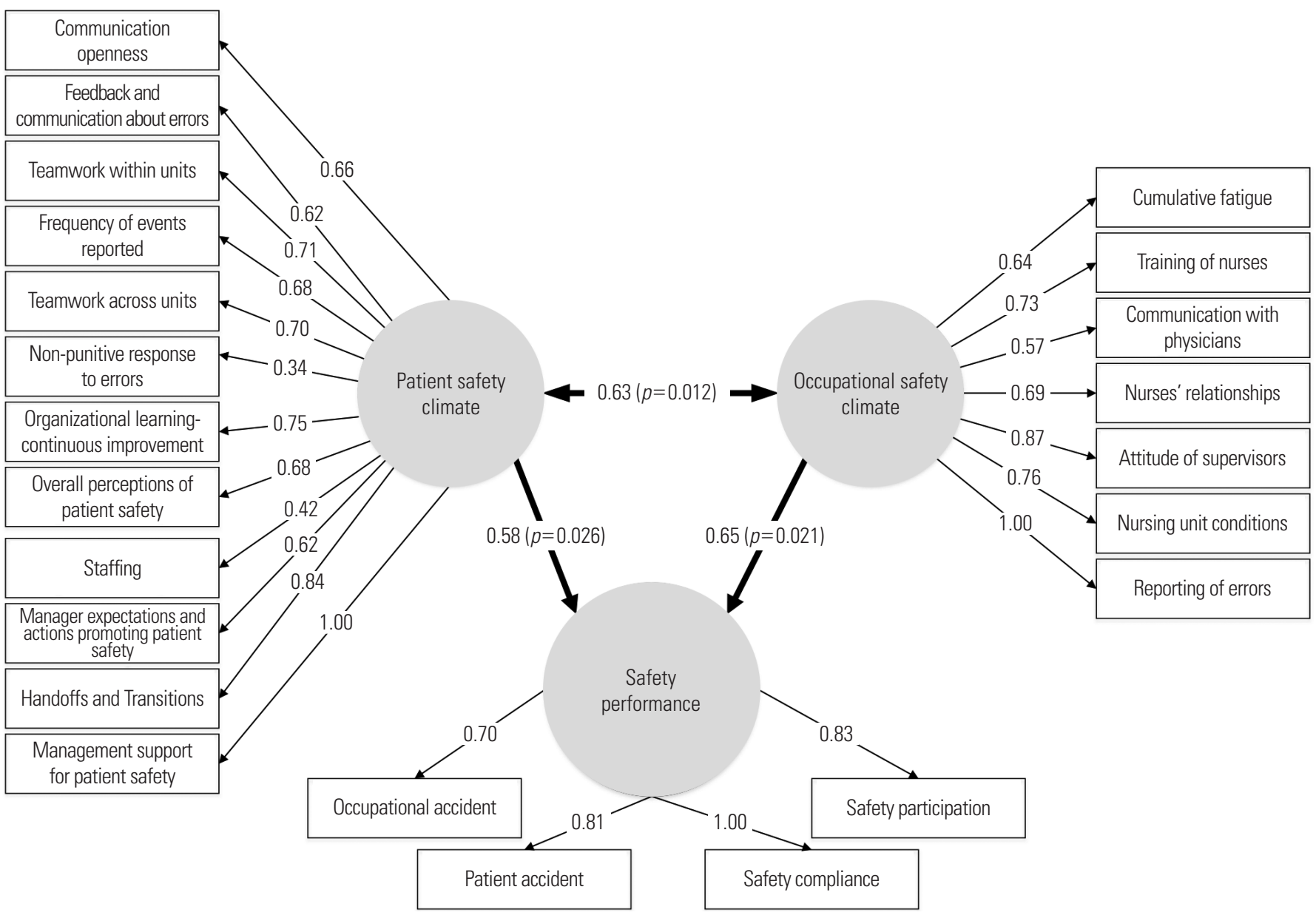

Figure 1. Causal relationship among occupational safety climate, patient safety climate, and safety performance.

(communication with physicians, relationship among nurses, cumulative fatigue, attitude of supervisors, nursing unit conditions, reporting of errors, and nursing training). The questionnaire consists of 29 questions scored on a 5-point Likert scale.

\section{Patient safety climate}

The patient safety climate was evaluated using the Iranian version of the Hospital Survey on Patient Safety Climate (HSOPSC) questionnaire, which was validated by Javad et al. [22]. The Agency for Health Care Research and Quality constructed the original HSOPSC questionnaire [23], which measures 10 dimensions of patient safety climate at the unit and hospital level and 2 outcomes. This questionnaire comprised 42 items scored on a 5 -point Likert scale.

\section{Safety performance}

To measure nurses' safety performance, a 5-point Likert scale questionnaire was constructed with 3 questions regard- ing safety compliance and 4 questions regarding safety participation. The questions of the questionnaire were translated into Persian. The validity of the questionnaire was evaluated in accordance with the opinion of 7 experts and Lawshe [24]'s method. Questions with a content validity ratio lower than 0.99 were deleted. Furthermore, the reliability of the questionnaire was assessed using the Cronbach alpha coefficient, which was more than 0.70 for all questions. It should be noted that adverse outcomes for nurses and patients were separately measured, using the following 2 items:

(1) Over the last 12 months, did you experience an occupational accident?; (2) Over the last 12 months, did you experience an action that led to an adverse outcome in patients?

\section{Statistical Analysis}

Structural equation modeling (SEM) is utilized to test the hypothetical model. SEM is a robust causal modeling approach that simultaneously estimates multiple and interrelat- 
ed dependencies between variables [25]. The goodness-of-fit of the model was assessed by indices including the comparative fit index (CFI), Tucker-Lewis index (TLI), $\chi^{2} / \mathrm{df}$, normed-fit index (NFI), and root mean square error of approximation (RMSEA) [26]. SPSS version 22.0 (IBM Corp., Armonk, NY, USA) was used for all analyses.

\section{Ethics Statement}

This study was approved by the Ethics Committee of Aja University (No. 91000227).

\section{RESULTS}

\section{Descriptive Results}

In total, 211 questionnaires were completed by the participants. As shown in Table 1, which presents the descriptive results and job characteristics, $43.0 \%$ of the studied participants were male, and $57.0 \%$ were female, $59.0 \%$ were married, and $84.0 \%$ had a bachelor's degree. Only $7.0 \%$ of participants had more than 15 years of work experience, and $57.5 \%$ were shift workers.

Table 2 shows the mean score for the occupational safety climate dimensions, patient safety climate dimensions, and

Table 1. General characteristics of the participants

\begin{tabular}{llr}
\hline Characteristics & Group & n (\%) \\
\hline Sex & Male & $91(43.0)$ \\
Marital status & Female & $120(57.0)$ \\
& Single & $80(38.0)$ \\
& Married & $125(59.0)$ \\
& Widowed & $3(1.5)$ \\
Age (y) & Divorced & $3(1.5)$ \\
& $20-30$ & $117(55.5)$ \\
& $30-40$ & $70(33.3)$ \\
Level of education & $40-50$ & $19(8.7)$ \\
& $>50$ & $5(2.5)$ \\
Job experience (y) & Diploma & $9(4.0)$ \\
& Bachelor's & $177(84.0)$ \\
& Master's & $25(12.0)$ \\
Shift type & $1-5$ & $109(51.5)$ \\
& $5-10$ & $45(21.5)$ \\
& $10-15$ & $42(20.0)$ \\
& $>15$ & $15(7.0)$ \\
& Morning & $55(26.0)$ \\
& Afternoon & $20(9.5)$ \\
& Night & $15(7.0)$ \\
& Shift work & $121(57.5)$ \\
\hline
\end{tabular}

safety performance dimensions. As shown in Table 2, the maximum and minimum mean scores of occupational safety climate dimensions were found for reporting of errors and cumulative fatigue, respectively. Among the dimensions of patient safety climate, the highest mean score was found for non-punitive response to errors, while the lowest mean score was found for manager expectations and actions promoting patient safety. The findings for safety performance dimensions showed that the mean scores for safety compliance and safety participation were $3.53 \pm 0.88$ and $3.69 \pm 0.75$, respectively.

Table 3 presents the findings of occupational and patient adverse outcomes. As observed, $67.5 \%$ of the nurses had experienced an occupational accident, and $32.5 \%$ of them had experienced adverse outcomes related to patients in the last 12 months. The most frequently reported occupational accidents were attributed to needlestick injuries (66.6\%), and medication errors (48.7\%) were the most frequently reported patient accidents.

Figure 1 shows the causal relationships among occupational

Table 2. Descriptive results of occupational safety climate and patient safety climate

\begin{tabular}{ll}
\hline Variables & Mean \pm SD \\
\hline Occupational safety climate dimensions & \\
Cumulative fatigue & $2.43 \pm 1.14$ \\
Training of nurses & $3.20 \pm 0.82$ \\
Communication with physicians & $3.09 \pm 0.85$ \\
Nurses' relationships & $3.52 \pm 0.85$ \\
Attitude of supervisors & $3.27 \pm 0.95$ \\
Nursing unit conditions & $2.96 \pm 0.89$ \\
Reporting of errors & $3.56 \pm 0.82$ \\
Patient safety climate dimensions & \\
Communication openness & $2.96 \pm 0.78$ \\
Feedback and communication about errors & $3.16 \pm 0.80$ \\
Teamwork within units & $3.22 \pm 0.97$ \\
Frequency of events reported & $3.17 \pm 0.88$ \\
Teamwork across units & $3.18 \pm 0.74$ \\
Non-punitive response to errors & $3.71 \pm 0.89$ \\
Organizational learning - continuous improvement & $3.12 \pm 0.90$ \\
Overall perceptions of patient safety & $3.18 \pm 0.73$ \\
Staffing & $3.27 \pm 0.82$ \\
Manager expectations and actions promoting patient safety & $2.86 \pm 0.89$ \\
Handoffs and transitions & $2.97 \pm 0.93$ \\
Management support for patient safety & $3.30 \pm 0.73$ \\
Safety performance dimensions & \\
Safety compliance & $3.53 \pm 0.88$ \\
Safety participation & $3.69 \pm 0.75$ \\
\hline
\end{tabular}

SD, standard deviation. 
Table 3. Findings on occupational and patient adverse outcomes

\begin{tabular}{llc}
\hline Variables & \multicolumn{1}{c}{ States } & n (\%) \\
\hline Occupational accident & Yes & $69(32.5)$ \\
& No & $142(67.5)$ \\
Type of occupational & Needlestick & $46(66.6)$ \\
accident & Exposure to chemical agents & $7(9.5)$ \\
& Exposure to biological agents & $16(23.9)$ \\
Patient accident & Yes & $49(23.0)$ \\
& No & $162(77.0)$ \\
Type of patient accident & Medication error & $24(48.7)$ \\
& Patient fall & $9(17.3)$ \\
& Pressure ulcer & $1(3.5)$ \\
& Physical restraints & $1(3.5)$ \\
& Infusion or transfusion reaction & $10(20.0)$ \\
& Patient complaints & $4(7.0)$ \\
\hline
\end{tabular}

safety climate, patient safety climate, and safety performance. As can be seen in Figure 1, the factors of management support for patient safety and handoffs and transition had the strongest influence on the patient safety climate. Reporting of errors and attitude of supervisors were the factors that showed the most influence on the occupational safety climate.

\section{Analytical Results}

The results of the hypothetical and causal model based on an evaluation of the relationships among occupational safety climate, patient safety climate, and safety performance are presented in Figure 1. These findings showed that all variables had a significant and direct effect on the relevant dimensions $(p<0.01)$. Moreover, the correlation coefficients were estimated to be greater than 0.50 . The correlation coefficient between occupational safety climate and patient safety climate was 0.63 , which was statistically significant $(p<0.05)$. The relationships of occupational safety climate and patient safety climate with safety performance were also significant, with correlation coefficients of 0.58 and 0.65 , respectively. The assessment of the goodness-of-fit in the structural causal analysis of the hypothetical model showed values of $\chi^{2} / \mathrm{df}$, RMSEA, CFI, NFI, TLI, and GFI indices of $1.85,0.077,0.89,0.82,0.86$, and 0.81 , respectively, confirming that the model was acceptable.

\section{DISCUSSION}

The present study was conducted to investigate the relationships among patient safety climate, occupational safety climate, and safety performance in hospitals. Previous studies $[8,9]$ have reported that unsafe behavior by nurses is one of the most important causes of injury in patients. Nurses' behavior has also been reported to have close relationships with occupational safety climate and patient safety climate [18]. However, no study has yet investigated both the occupational safety climate and patient safety climate as important factors contributing to the safe behavior of nurses and healthcare staff.

According to our findings, among the dimensions of occupational safety climate, reporting of errors and cumulative fatigue had the highest and the lowest mean values, respectively. Zarei et al. [21] likewise reported that cumulative fatigue had the lowest mean score among all dimensions of occupational safety climate. However, occupational fatigue among hospital staff is an important issue that should not be underestimated. It can be affected by many factors, such as workload, sleep quality, overtime, and the social environment in the workplace $[27,28]$. Furthermore, it can affect safety outcomes such as needlestick and sharp injuries, human error, and injuries [27,29]. Zarei et al. [21] found that nurses' relationships had the highest mean score, but in the present study, the highest scores were found for non-punitive response to errors and management support for patient safety had the highest mean values. This result is consistent with the findings of Pousette et al. [19] that hospital management support for patient safety had the lowest mean score. Management support is necessary for the successful implementation of any program in an organization. Therefore, it can be concluded that appropriate and practical management support is needed to patient safety in hospitals [30]. There are several ways in which management can demonstrate support for safety, including resource allocation, adopting appropriate policies, and proper error management.

Safety performance can be measured by either reactive or proactive indicators [31]. In this study, safety performance was measured using 2 reactive indices (including occupational accidents and patient accidents) and 2 proactive indicators (safety compliance and safety participation). The safety performance measurements revealed that these 4 reactive and proactive dimensions were suitable for assessing this factor. Safety compliance and safety participation had nearly equal mean scores.

The results of this study showed that needlestick injuries the most common occupational accident in healthcare units, and medication errors were a common problem in relation to patient safety. The results of the current study are consistent with 
the findings of several previous studies [29,32]. Significant relationships were found between both types of safety climate and safety performance; therefore, improvements in occupational safety climate and patient safety climate will improve the safety performance of nurses, thereby helping to prevent needlestick injuries.

As expected, medication error was the most prevalent type of accident related to patients. A variety of factors at different organizational levels can affect such errors [33]. Hospitals should put in place a proper error management program for recording, analyzing, and preventing medication errors. Patient falls were another common type of accident, and were frequently encountered among disabled patients (physically or mentally) and elderly. As patient falls increase treatment costs and prolong hospital stays, they should be controlled using available technologies and well-designed training programs [34].

The SEM approach in the present study was employed to evaluate the relationships among occupational safety climate, patient safety climate, and safety performance in nurses. The results revealed a significant positive association between the occupational safety climate and patient safety climate. This result is in agreement with the findings of Pousette et al. [19], who showed a strong association between occupational safety climate and patient safety domains. This finding may have occurred because these constructs have many aspects in common. For example, nurses' relationships, as a dimension of the occupational safety climate, is very similar to teamwork as a dimension of patient safety climate. In other words, good teamwork requires good relationships among nurses. Likewise, reporting of errors, as a dimension of the occupational safety climate, is closely related to non-punitive response to error, because when managers punish employees for their unintentional errors, employees might be afraid of reporting their errors.

We found direct and significant relationships between both types of safety climate with safety performance. Therefore, improving both types of safety climate will result in improvements in nurses' safety performance. Cooper and Phillips [35] reported that enhancing the safety climate can provide an opportunity to improve safety performance. Therefore, these findings have multilateral implications, offering ways to improve the quality of hospital and care services, the job satisfaction of nurses, and patient satisfaction. Agnew et al. [9] showed a significant correlation between the patient safety climate and clinical workers' safety behavior. Moreover, multiple studies $[36,37]$ have found positive relationships between the occupational safety climate and safety performance. In this study, there was a close correlation between both types of safety climate, so improving one of them is likely to enhance the other. For instance, enhancing the patient safety climate requires special attention to human error issues. In this regard, error reporting mechanisms should be facilitated, which is a dimension of the safety climate.

This study has some limitations that should be taken into consideration, such as the limited number of participants. Longitudinal studies are also recommended to better address causal relationships among the variables.

The results of the present study indicate that the occupational safety climate, patient safety climate, and nurses' safety performance were closely correlated. Therefore, improving the occupational and/or patient safety climate can lead to improvements in the nurses' safety performance of nurses. Improvement of safety performance can lead to fewer occupational accidents and improve the quality of health care units and patients' satisfaction. Furthermore, it is recommended to improve the quality of each of the 7 dimensions of occupational safety climate and the 12 dimensions of patient safety climate by implementing appropriate measures.

\section{CONFLICT OF INTEREST}

The authors have no conflicts of interest associated with the material presented in this paper.

\section{FUNDING}

This work was supported by research grants from Aja University of Medical Sciences (grant No. 91000227).

\section{ACKNOWLEDGEMENTS}

The authors would like to thank all nurses for their kind cooperation with the researchers in collecting the data.

\section{AUTHOR CONTRIBUTIONS}

Conceptualization: HA, ZSA. Data curation: HA. Formal analysis: MMA, HA. Funding acquisition: HA. Methodology: HA, ZSA, MMA, HA. Project administration: ZSA. Visualization: HA, HA. Writing - original draft: HA, ZSA. Writing - review \& editing: $\mathrm{HA}, \mathrm{MMA}$. 


\section{ORCID}

Hamed Aghaei https://orcid.org/0000-0003-3283-2066

Zahra Sadat Asadi https://orcid.org/0000-0002-9193-046X

Mostafa Mirzaei Aliabadi https://orcid.org/0000-0003-37726780

Hassan Ahmadinia https://orcid.org/0000-0002-7010-1726

\section{REFERENCES}

1. Owens ML. The effect of safety climate, teamwork, and sustainable motivation on nurse job satisfaction and intent to stay [dissertation]. Tyler: University of Texas at Tyler; 2011.

2. Gershon RR, Stone PW, Zeltser M, Faucett J, MacDavitt K, Chou SS. Organizational climate and nurse health outcomes in the United States: a systematic review. Ind Health 2007;45(5):622636.

3. World Health Organization. Patient safety; 2019 [cited 2020 Jul 1]. Available from: https://www.who.int/news-room/factsin-pictures/detail/patient-safety.

4. Zohar D. Safety climate in industrial organizations: theoretical and applied implications. J Appl Psychol 1980;65(1):96-102.

5. Kudo Y, Kido S, Taruzuka Shahzad M, Saegusa Y, Satoh T, Aizawa Y. Safety climate and motivation toward patient safety among Japanese nurses in hospitals of fewer than 250 beds. Ind Health 2009;47(1):70-79.

6. Lin SH, Tang WJ, Miao JY, Wang ZM, Wang PX. Safety climate measurement at workplace in China: a validity and reliability assessment. Saf Sci 2008;46(7):1037-1046.

7. Najjar S, Nafouri N, Vanhaecht K, Euwema M. The relationship between patient safety culture and adverse events: a study in Palestinian hospitals. Saf Health 2015;1(1):16.

8. Kohn LT, Janet Corrigan J, Donaldson MS; Committee on Quality of Health Care in America. To err is human: building a safer health system. Washington, DC: National Academy Press; 1999, p. 18-24.

9. Agnew C, Flin R, Mearns K. Patient safety climate and worker safety behaviours in acute hospitals in Scotland. J Safety Res 2013;45:95-101.

10. Weaver SJ, Lubomksi LH, Wilson RF, Pfoh ER, Martinez KA, Dy SM. Promoting a culture of safety as a patient safety strategy: a systematic review. Ann Intern Med 2013;158(5 Pt 2):369-374.

11. Mirzaei Aliabadi M, Aghaei H, Kalatpour O, Soltanian AR, Nikravesh A. Analysis of human and organizational factors that influence mining accidents based on Bayesian network. Int J
Occup Saf Ergon 2018:1-8.

12. Lyu S, Hon CK, Chan AP, Wong FK, Javed AA. Relationships among safety climate, safety behavior, and safety outcomes for ethnic minority construction workers. Int J Environ Res Public Health 2018;15(3):484.

13. Neal A, Griffin MA, Hart PM. The impact of organizational climate on safety climate and individual behavior. Saf Sci 2000; 34(1-3):99-109.

14. Siu OL, Phillips DR, Leung TW. Safety climate and safety performance among construction workers in Hong Kong. The role of psychological strains as mediators. Accid Anal Prev 2004;36(3):359-366.

15. Pousette A, Larsson S, Törner M. Safety climate cross-validation, strength and prediction of safety behaviour. Saf Sci 2008;46(3): 398-404.

16. Hon CK, Chan AP, Yam MC. Relationships between safety climate and safety performance of building repair, maintenance, minor alteration, and addition (RMAA) works. Saf Sci 2014;65: 10-19.

17. Kuenzi $M$, Schminke $M$. Assembling fragments into a lens: a review, critique, and proposed research agenda for the organizational work climate literature. J Manag 2009;35(3):634-717.

18. Flin R. Measuring safety culture in healthcare: a case for accurate diagnosis. Saf Sci 2007;45(6):653-667.

19. Pousette A, Larsman $P$, Eklöf $M$, Törner M. The relationship between patient safety climate and occupational safety climate in healthcare - a multi-level investigation. J Safety Res 2017; 61:187-198.

20. McCaughey D, DelliFraine JL, McGhan G, Bruning NS. The negative effects of workplace injury and illness on workplace safety climate perceptions and health care worker outcomes. Saf Sci 2013;51(1):138-147.

21. Zarei E, Khakzad N, Reniers G, Akbari R. On the relationship between safety climate and occupational burnout in healthcare organizations. Saf Sci 2016;89:1-10.

22. Javad M, Amin G, Mahbobe M, Forooshani R, Abas AS, Mohammad A. Validation of Farsi version of hospital survey on patient safety culture questionnaire, using confirmatory factor analysis method. Hosp J 2012;11(2):19-30 (Persian).

23. Nieva VF, Sorra J. Safety culture assessment: a tool for improving patient safety in healthcare organizations. BMJ Qual Saf 2003;12(Suppl 2):ii17-ii23.

24. Lawshe $\mathrm{CH}$. A quantitative approach to content validity. Pers Psychol 1975;28(4):563-575.

25. Mirzaei Aliabadi M, Aghaei H, Kalatpour O, Soltanian AR, Seyed- 
Tabib M. Effects of human and organizational deficiencies on workers' safety behavior at a mining site in Iran. Epidemiol Health 2018;40:e2018019.

26. Soltanzadeh A, Mohammadfam I, Moghimbeygi A, Ghiasvand R. Exploring causal factors on the severity rate of occupational accidents in construction worksites. Int J Civ Eng 2017;15(7): 959-965.

27. Techera U, Hallowell M, Stambaugh N, Littlejohn R. Causes and consequences of occupational fatigue: meta-analysis and systems model. J Occup Environ Med 2016;58(10):961-973.

28. Ghasemi F, Samavat P, Soleimani F. The links among workload, sleep quality, and fatigue in nurses: a structural equation modeling approach. Fatigue 2019;7(3):141-152.

29. Akbari H, Ghasemi F, Akbari H, Adibzadeh A. Predicting needlestick and sharps injuries and determining preventive strategies using a Bayesian network approach in Tehran, Iran. Epidemiol Health 2018;40:e2018042.

30. Feng XQ, Acord L, Cheng YJ, Zeng JH, Song JP. The relationship between management safety commitment and patient safety culture. Int Nurs Rev 2011;58(2):249-254.

31. Di Gravio G, Mancini M, Patriarca R, Costantino F. Overall safety performance of the air traffic management system: indica- tors and analysis. J Air Transp Manag 2015;44:65-69.

32. Antony J, Forthun SC, Trakulsunti Y, Farrington T, McFarlane J, Brennan A, et al. An exploratory study into the use of Lean Six Sigma to reduce medication errors in the Norwegian public healthcare context. Leadersh Health Serv (Bradf Engl) 2019; 32(4):509-524.

33. Aspden P, Wolcott JA, Bootman JL, Cronenwett LR. Preventing medication errors: quality chasm series. Washington, DC: National Academy Press; 2007, p. 409-446.

34. Chaccour K, Darazi R, El Hassani AH, Andres E. From fall detection to fall prevention: a generic classification of fall-related systems. IEEE Sens J 2016;17(3):812-822.

35. Cooper MD, Phillips RA. Exploratory analysis of the safety climate and safety behavior relationship. J Safety Res 2004;35(5): 497-512.

36. Tholén SL, Pousette A, Törner M. Causal relations between psychosocial conditions, safety climate and safety behavioura multi-level investigation. Saf Sci 2013;55:62-69.

37. Clarke $S$. The relationship between safety climate and safety performance: a meta-analytic review. J Occup Health Psychol 2006;11(4):315-327. 